Research Paper

\title{
Anemia as an Independent Predictor of Adverse Cardiac Outcomes in Patients with Atrial Fibrillation
}

\author{
Wen-Hsien Lee ${ }^{1,2,3}$, Po-Chao Hsu ${ }^{1,3}$, Chun-Yuan Chu ${ }^{1}$, Hung-Hao Lee ${ }^{1}$, Meng-Kuang Lee ${ }^{1,2}$, Chee-Siong \\ Lee $^{1,3}$, Hsueh-Wei Yen 1,3 , Tsung-Hsien Lin ${ }^{1,3}$, Wen-Chol Voon ${ }^{1,3}$, Wen-Ter Lai ${ }^{1,3}$, Sheng-Hsiung Sheu ${ }^{1,3}$, \\ Ho-Ming Su1,2,3凶 \\ 1. Division of Cardiology, Department of Internal Medicine, Kaohsiung Medical University Hospital, Kaohsiung Medical University, \\ Kaohsiung, Taiwan \\ 2. Department of Internal Medicine, Kaohsiung Municipal Hsiao-Kang Hospital, Kaohsiung Medical University, Kaohsiung, Taiwan \\ 3. Faculty of Medicine, College of Medicine, Kaohsiung Medical University, Kaohsiung, Taiwan
}

$\triangle$ Corresponding author: Ho-Ming Su, MD, Department of Internal Medicine, Kaohsiung Municipal Hsiao-Kang Hospital, Kaohsiung Medical University, 482, Shan-Ming Rd., Hsiao-Kang Dist., 812 Kaohsiung, Taiwan. TEL: 886- 7- 8036783 - 3441; FAX: 886- 7- 8063346; E-mail: cobeshm@seed.net.tw

(C) 2015 Ivyspring International Publisher. Reproduction is permitted for personal, noncommercial use, provided that the article is in whole, unmodified, and properly cited. See http://ivyspring.com/terms for terms and conditions.

Received: 2015.02.17; Accepted: 2015.06.09; Published: 2015.07.16

\begin{abstract}
Background: Anemia and echocardiographic systolic and diastolic parameters are useful predictors of cardiovascular outcomes in patients with atrial fibrillation (AF). However, no studies have evaluated the use of anemia for predicting cardiovascular outcome in AF patients when the important echocardiographic parameters are known. Therefore, this study was designed to evaluate whether low hemoglobin is a useful parameter for predicting poor cardiac outcome after adjustment for important echocardiographic parameters in AF patients.

Methods: Index beat method was used to measure echocardiographic parameters in 166 patients with persistent AF. Cardiac events were defined as death and hospitalization for heart failure. The association of hemoglobin with adverse cardiac events was assessed by Cox proportional hazards model.

Results: The 49 cardiac events identified in this population included 21 deaths and 28 hospitalizations for heart failure during an average follow-up of 20 months $\left(25^{\text {th }}-75^{\text {th }}\right.$ percentile: $14-32$ months). Multivariable analysis showed that increased left ventricular mass index (LVMI) and decreased body mass index, estimated glomerular filtration rate, and hemoglobin (hazard ratio 0.827; $P=0.015)$ were independently associated with increased cardiac events. Additionally, tests of a Cox model that included important clinic variables, LVMI, left ventricular ejection fraction, and the ratio of transmitral E-wave velocity to early diastolic mitral annulus velocity showed that including hemoglobin significantly increased value in predicting adverse cardiac events $(P=0.010)$.

Conclusions: Hemoglobin is a useful parameter for predicting adverse cardiac events, and including hemoglobin may improve the prognostic prediction of conventional clinical and echocardiographic parameters in patients with AF.
\end{abstract}

Key words: Hemoglobin, anemia, atrial fibrillation, cardiac outcomes.

\section{Introduction}

Atrial fibrillation (AF) is the most common form of cardiac arrhythmia in adults. Its prevalence increases with age and reportedly reaches $9 \%$ in those older than 80 years [1]. Patients with AF often have other cardiovascular comorbidities, including chronic heart failure, stroke, valvular heart disease, hypertension, and diabetes mellitus [2]. AF is independently associated with increased risks of ischemic stroke, 
hospitalization for heart failure, and mortality [3-5]. Cardiovascular comorbidities and thromboembolic events significantly increase the mortality rate and treatment cost of AF [6].

Anemia, which is defined as a reduced hemoglobin concentration or hematocrit, is among the most common disorders in the world and is a major public health concern in both industrialized and non-industrialized countries. Globally, anemia affects about 1.62 billion people, which corresponds to $24.8 \%$ of the overall population [7]. Anemia, which is a risk factor for cardiovascular disease, is independently associated with an increased mortality rate in patients with chronic heart failure, left ventricular hypertrophy, chronic kidney disease, diabetes mellitus, and acute coronary syndrome [8-12]. Several studies have investigated the relationship between cardiovascular outcome and anemia in patients with AF. For example, an analysis of 3378 Japanese AF patients enrolled in the Fushimi AF Registry indicated that, compared to patients who had AF alone, patients who had both $\mathrm{AF}$ and anemia had more clinical comorbidities, including old age, heart failure, coronary artery disease, peripheral artery disease, chronic kidney disease, and stroke [13]. Sharma et al. reported that anemia was an independent predictor of mortality and hospitalizations in 13067 elderly patients with AF in the United States [14]. In elderly AF patients, low hematocrit is also associated with an increased mortality rate. Additionally, echocardiographic parameters, including left ventricular hypertrophy and left ventricular diastolic and systolic dysfunction, are well-established predictors of cardiovascular outcomes in patients irrespective of the presence of AF [15, 16,17-23]. However, no study has investigated the incremental value of anemia for predicting cardiovascular outcome in AF patients when important clinical and echocardiographic parameters are known. Therefore, this study investigated whether low hemoglobin is a useful parameter for predicting poor cardiac outcome and whether including anemia with the clinical and echocardiographic parameters conventionally used to predict adverse cardiac events in AF patients further improves predictive value.

\section{Methods}

\section{Study patients}

This prospective observational cohort study included patients with persistent AF referred for echocardiographic examinations at Kaohsiung Municipal Hsiao-Kang Hospital from April, 2010 to June, 2012. Persistent AF was defined as AF lasting for at least 7 days according to 12-lead eletrocardiography (ECG), 24-hour Holter ECG, or ECG during echocardio- graphic examination. Patients were excluded if they had inadequate echocardiographic visualization and a major valvular heart disease (i.e., moderate/severe mitral stenosis, moderate/severe aortic stenosis or regurgitation, or severe mitral regurgitation). Patients were also excluded if they had acute or chronic bleeding and deficiency of vitamin B12, folate, or iron. The final population included 166 AF patients. The study protocol was approved by the Institutional Review Board of Kaohsiung Municipal Hsiao-Kang Hospital, and all enrolled patients gave written, informed consent to participate in the study.

\section{Echocardiographic evaluation}

Echocardiographic examinations were performed with a VIVID 7 (General Electric Medical Systems, Horten, Norway) with the participant respiring quietly in the left decubitus position. All examinations were performed by one experienced cardiologist who was blinded to all clinical data, including history of hypertension, diabetes mellitus, coronary artery disease, etc. Two-dimensional and anatomic M-mode images were recorded in standardized views. The Doppler sample volume was placed at the tips of the mitral leaflets to obtain the left ventricular inflow waveforms in apical 4-chamber view. Pulsed tissue Doppler imaging was obtained with the sample volume placed at the lateral and septal corners of the mitral annulus in apical 4-chamber view. Early diastolic mitral annulus velocity (Ea) was obtained by averaging septal and lateral velocities. The wall filter settings were adjusted to exclude high-frequency signals, and the gain was minimized. Left ventricular ejection fraction (LVEF) was measured using the modified Simpson method. Left ventricular mass was calculated using Devereux-modified method [24]. Left ventricular mass index (LVMI) was calculated by dividing left ventricular mass by body surface area. Left atrial volume was measured using the biplane area-length method [25]. Left atrial volume index (LAVI) was calculated by dividing left atrial volume by body surface area.

The LVEF, LAVI, and LVMI were measured from the index beat [26-28]. Since the early mitral inflow velocity (E), E-wave deceleration time, and Ea could be obtained quickly and easily, they were obtained from five beats and then averaged for later analysis [29]. If the cardiac cycle length was too short to complete the diastolic process, this beat was skipped. Thus, the selection of E, E-wave deceleration time and Ea was not always consecutive. Heart rate was obtained from five consecutive beats. The raw ultrasonic data, including 15 consecutive beats from apical 4-chamber and 2-chamber views, were recorded and analyzed offline using EchoPAC software 
(EchoPAC version 08; GE-Vingmed Ultrasound AS GE Medical Systems).

\section{Index beat selection}

The index beat was taken after two approximately equal preceding and pre-preceding intervals selected from 15 stored cardiac cycles. The index beat was defined as if both preceding and pre-preceding intervals of the index beat were $>500 \mathrm{~ms}$ [30] and if the difference between the two intervals was less than 60 $\mathrm{ms}$ [31]. The criterion for the cardiac cycle of the index beat was also $>500 \mathrm{~ms}$ [30]. The patient was excluded if no beat in the 15 stored cardiac cycles met the required index beat. If several beats in the 15 stored cardiac cycles met the criteria for the index beat, the first index beat was used to calculate the echocardiographic data.

\section{Collection of demographic, medical, and laboratory data}

Demographic and medical data were obtained from medical records or from interviews with patients and included age, gender, and any history of diabetes mellitus, hypertension, coronary artery disease, stroke or chronic heart failure. The body mass index was calculated as the ratio of weight in kilograms divided by the square of height in meters. The systolic and diastolic blood pressures were measured by mercury sphygmomanometer before echocardiographic examination. Diabetes mellitus was defined as a fasting blood glucose level higher than $126 \mathrm{mg} / \mathrm{dL}$ or prescription for hypoglycemic agents to control blood glucose levels. Similarly, hypertension was defined as systolic blood pressure $\geq 140 \mathrm{mmHg}$ or diastolic blood pressure $\geq 90 \mathrm{mmHg}$ or prescription for anti-hypertensive drugs. Stroke was defined as any history of cerebrovascular accident, including cerebral bleeding and infarction. Coronary artery disease was defined as any history of typical angina with positive stress test, angiographically documented coronary artery disease, myocardial infarction, coronary artery bypass surgery, or angioplasty. Heart failure was defined according to Framingham criteria. Laboratory data collection included total cholesterol and triglyceride. Medical records were reviewed for history of medications during the study period, including use of angiotensin converting enzyme inhibitors, angiotensin II receptor blockers, $\beta$-blockers, calcium channel blockers, diuretics, antiplatelet drugs, and anticoagulant drugs. According to the World Health Organization definition, anemic patients were defined as hemoglobin level less than $12 \mathrm{~g} / \mathrm{dL}$ for women and less than $13 \mathrm{~g} / \mathrm{dL}$ for men [32].

\section{Definition of cardiac events}

Cardiac events were defined as all-cause mortal- ity and hospitalization for heart failure. Hospitalization for heart failure was defined as admission due to dyspnea with chest radiographic evidence of pulmonary congestion and treatment with intravenous diuretics. Cardiac events were ascertained and adjudicated by two cardiologists based on the course of hospital treatment indicated in the medical record. In the case of a disagreement, a third cardiologist defined the cardiac event. If a patient had multiple cardiac events, only the first event was coded. However, the death of a patient after a heart failure episode during the same admission was coded as a death. Patients who reached the study endpoints were followed up until the first adverse event. All other patients were followed up until May, 2013.

\section{Statistical analysis}

The SPSS 18.0 software (SPSS, Chicago, IL, USA) was used for statistical analysis. Continuous and categorical variables were compared between groups by independent sample t-test and by Chi-square test, respectively. The significant variables in the univariable analysis were selected for multivariable analysis. Time to adverse events and covariates of risk factors were modeled using a Cox proportional hazards model. Incremental model performance was assessed by a change in the Chi-square value. Kaplan-Meier survival plots were calculated from baseline to the time of an adverse event and compared by Log-rank test. All tests were 2-sided, and a P value less than 0.05 was considered statistically significant.

\section{Results}

For the 166 patients in this study, the mean age and the mean serum hemoglobin value were $71.0 \pm$ 10.0 years and $13.4 \pm 2.2 \mathrm{~g} / \mathrm{dL}$, respectively. Table 1 compares the clinical and echocardiographic characteristics between anemic and non-anemic patients. The two groups significantly differed in age, history of coronary artery disease, history of congestive heart failure, diastolic blood pressure, total cholesterol, estimated glomerular filtration rate, hemoglobin, use of $\beta$-blockers, LAVI, LVMI, E, Ea, and E/Ea.

For all patients, the follow-up period to cardiac events was 20 months $\left(25^{\text {th }}-75^{\text {th }}\right.$ percentile: $14-32$ months). Forty-nine cardiac events were documented during the follow-up period, including 21 deaths and 28 hospitalizations for heart failure. Table 2 shows the results of a Cox proportional hazards regression analysis of cardiac events. Univariable analysis of adverse cardiac events revealed significant associations with old age, presence of chronic heart failure, diuretic use, decreased body mass index, estimated glomerular filtration rate, hemoglobin (hazard ratio [HR] 0.789; 95\% confidence interval [CI] 0.700 to 0.890 ; 
$\mathrm{P}<0.001)$, LVEF, and Ea, and increased LVMI, E, E-wave deceleration time, and E/Ea were significantly related to adverse cardiac events. In the multivariable analysis, increased cardiac events had sig- nificant independent associations with increased LVMI, decreased body mass index, estimated glomerular filtration rate, and hemoglobin (HR 0.827; $95 \%$ CI 0.709 to $0.964 ; \mathrm{P}=0.015$ ).

Table 1. Comparison of clinical and echocardiographic characteristics between anemic and non-anemic patients

\begin{tabular}{|c|c|c|c|c|}
\hline Characteristics & $\begin{array}{l}\text { Anemic patients } \\
(\mathrm{n}=54)\end{array}$ & $\begin{array}{l}\text { Non-anemic patients } \\
(n=112)\end{array}$ & $P$ value & $\begin{array}{l}\text { All patients } \\
(\mathrm{n}=166)\end{array}$ \\
\hline Age (years) & $74.4 \pm 8.0$ & $69.4 \pm 10.4$ & 0.003 & $71.0 \pm 10.0$ \\
\hline Male (\%) & 68.5 & 67.9 & 0.932 & 68.1 \\
\hline Diabetes mellitus (\%) & 34.0 & 25.9 & 0.284 & 28.5 \\
\hline Hypertension (\%) & 63.0 & 66.1 & 0.694 & 65.1 \\
\hline CAD $(\%)$ & 18.9 & 8.0 & 0.042 & 11.5 \\
\hline Stroke $(\%)$ & 15.1 & 19.6 & 0.479 & 18.2 \\
\hline $\mathrm{CHF}(\%)$ & 45.3 & 24.1 & 0.006 & 30.9 \\
\hline SBP (mmHg) & $129.5 \pm 21.1$ & $133.0 \pm 20.3$ & 0.355 & $131.9 \pm 20.5$ \\
\hline $\mathrm{DBP}(\mathrm{mmHg})$ & $71.6 \pm 12.5$ & $78.4 \pm 12.5$ & 0.004 & $76.1 \pm 12.8$ \\
\hline Heart rate $\left(\mathrm{min}^{-1}\right)$ & $83.1 \pm 21.2$ & $83.8 \pm 18.9$ & 0.833 & $83.6 \pm 19.6$ \\
\hline Body mass index $\left(\mathrm{kg} / \mathrm{m}^{2}\right)$ & $25.4 \pm 4.4$ & $26.3 \pm 3.9$ & 0.171 & $26.0 \pm 4.1$ \\
\hline Triglyceride (mg/dL) & $125.4 \pm 84.2$ & $118.0 \pm 74.4$ & 0.615 & $120.2 \pm 77.3$ \\
\hline Total cholesterol (mg/dL) & $154.9 \pm 33.3$ & $180.8 \pm 35.1$ & $<0.001$ & $172.9 \pm 36.5$ \\
\hline eGFR (mL/min/1.73 m²) & $45.6 \pm 21.0$ & $56.2 \pm 15.8$ & 0.001 & $53.0 \pm 18.1$ \\
\hline Hemoglobin (g/dL) & $11.0 \pm 1.4$ & $14.6 \pm 1.2$ & $<0.001$ & $13.4 \pm 2.2$ \\
\hline \multicolumn{5}{|l|}{ Medications } \\
\hline ACEI and/or ARB (\%) & 50.9 & 58.0 & 0.392 & 55.8 \\
\hline$\beta$-blocker $(\%)$ & 30.2 & 48.2 & 0.029 & 42.4 \\
\hline CCB $(\%)$ & 30.2 & 35.7 & 0.484 & 33.9 \\
\hline Diuretics (\%) & 52.8 & 38.4 & 0.080 & 43.0 \\
\hline Antiplatelet (\%) & 51.9 & 62.5 & 0.191 & 59.0 \\
\hline Anticoagulant (\%) & 25.9 & 33.9 & 0.298 & 31.3 \\
\hline \multicolumn{5}{|l|}{ Echocardiographic data } \\
\hline LAVI $\left(\mathrm{ml} / \mathrm{m}^{2}\right)$ & $52.5 \pm 21.1$ & $45.7 \pm 18.9$ & 0.047 & $47.9 \pm 19.8$ \\
\hline $\operatorname{LVMI}\left(\mathrm{g} / \mathrm{m}^{2}\right)$ & $149.7 \pm 37.7$ & $135.6 \pm 41.5$ & 0.041 & $140.2 \pm 40.7$ \\
\hline LVEF (\%) & $53.7 \pm 15.5$ & $54.5 \pm 14.1$ & 0.724 & $54.3 \pm 14.5$ \\
\hline $\mathrm{E}(\mathrm{cm} / \mathrm{s})$ & $106.5 \pm 23.8$ & $92.3 \pm 21.1$ & $<0.001$ & $96.8 \pm 22.9$ \\
\hline EDT (ms) & $156.5 \pm 48.3$ & $147.8 \pm 45.3$ & 0.270 & $150.6 \pm 46.3$ \\
\hline $\mathrm{Ea}(\mathrm{cm} / \mathrm{s})$ & $8.0 \pm 2.0$ & $9.0 \pm 2.5$ & 0.009 & $8.7 \pm 2.4$ \\
\hline E/Ea & $14.1 \pm 5.4$ & $11.0 \pm 4.0$ & $<0.001$ & $12.0 \pm 4.7$ \\
\hline
\end{tabular}

ACEI: angiotensin converting enzyme inhibitor; ARB: angiotensin II receptor blocker; CAD: coronary artery disease; CCB: calcium channel blocker; CHF: chronic heart failure; DBP: diastolic blood pressure; E: early mitral inflow velocity; eGFR: estimated glomerular filtration rate; Ea: early diastolic mitral annulus velocity; EDT: E wave deceleration time; LAVI: left atrial volume index; LVEF: left ventricular ejection fraction; LVMI: left ventricular mass index; SBP: systolic blood pressure.

Table 2. Predictors of cardiac events (all-cause mortality and hospitalization for heart failure) using Cox proportional hazards model

\begin{tabular}{|c|c|c|c|c|}
\hline Parameter & Univariable & & Multivariable & \\
\hline & HR $(95 \%$ CI) & $P$ value & HR (95\% CI) & $P$ value \\
\hline Age (years) & $1.042(1.010,1.074)$ & 0.009 & $1.019(0.975,1.064)$ & 0.406 \\
\hline Male versus female & $0.858(0.477,1.546)$ & 0.611 & - & \\
\hline Diabetes mellitus & $1.259(0.685,2.315)$ & 0.458 & - & \\
\hline Hypertension & $1.419(0.806,2.499)$ & 0.226 & - & \\
\hline CAD (\%) & $0.892(0.400,1.989)$ & 0.780 & - & \\
\hline Stroke (\%) & $1.121(0.503,2.501)$ & 0.780 & - & \\
\hline CHF (\%) & $3.475(1.976,6.111)$ & $<0.001$ & $1.429(0.656,3.112)$ & 0.368 \\
\hline SBP (mmHg) & $1.004(0.988,1.020)$ & 0.658 & - & \\
\hline DBP (mmHg) & $1.005(0.980,1.031)$ & 0.676 & - & \\
\hline Heart rate $\left(\mathrm{min}^{-1}\right)$ & $1.006(0.992,1.021)$ & 0.377 & - & \\
\hline Body mass index $\left(\mathrm{kg} / \mathrm{m}^{2}\right)$ & $0.904(0.835,0.978)$ & 0.012 & $0.911(0.833,0.998)$ & 0.045 \\
\hline Triglyceride (mg/dL) & $1.000(0.995,1.004)$ & 0.855 & - & \\
\hline Total cholesterol (mg/dL) & $0.999(0.990,1.008)$ & 0.842 & - & \\
\hline eGFR (mL/min/1.73 m²) & $0.974(0.958,0.990)$ & 0.002 & $0.986(0.969,1.002)$ & 0.092 \\
\hline Hemoglobin $(\mathrm{g} / \mathrm{dL})$ & $0.789(0.700,0.890)$ & $<0.001$ & $0.827(0.709,0.964)$ & 0.015 \\
\hline \multicolumn{5}{|l|}{ Medications } \\
\hline ACEI and/or ARB (\%) & $1.119(0.632,1.978)$ & 0.700 & - & \\
\hline$\beta$-blocker (\%) & $0.915(0.519,1.614)$ & 0.760 & - & \\
\hline CCB $(\%)$ & $0.844(0.468,1.521)$ & 0.572 & - & \\
\hline Diuretics (\%) & $2.767(1.536,4.984)$ & 0.001 & $1.844(0.916,3.713)$ & 0.086 \\
\hline Antiplatelet (\%) & $1.143(0.651,2.008)$ & 0.641 & - & \\
\hline
\end{tabular}




\begin{tabular}{|c|c|c|c|c|}
\hline Anticoagulant (\%) & $0.935(0.502,1.738)$ & 0.831 & - & \\
\hline \multicolumn{5}{|l|}{ Echocardiographic data } \\
\hline LAVI $\left(\mathrm{ml} / \mathrm{m}^{2}\right)$ & $1.009(0.996,1.022)$ & 0.156 & - & \\
\hline $\operatorname{LVMI}\left(\mathrm{g} / \mathrm{m}^{2}\right)$ & $1.013(1.006,1.020)$ & $<0.001$ & $1.009(1.001,1.018)$ & 0.028 \\
\hline LVEF (\%) & $0.962(0.944,0.981)$ & $<0.001$ & $0.991(0.961,1.023)$ & 0.590 \\
\hline $\mathrm{E}(\mathrm{cm} / \mathrm{s})$ & $1.014(1.003,1.026)$ & 0.017 & $1.023(0.996,1.051)$ & 0.092 \\
\hline EDT (ms) & $1.006(1.000,1.011)$ & 0.036 & $0.999(0.992,1.006)$ & 0.815 \\
\hline $\mathrm{Ea}(\mathrm{cm} / \mathrm{s})$ & $0.727(0.637,0.830)$ & $<0.001$ & $0.762(0.524,1.107)$ & 0.154 \\
\hline $\mathrm{E} / \mathrm{Ea}$ & $1.116(1.072,1.162)$ & $<0.001$ & $0.959(0.813,1.132)$ & 0.622 \\
\hline
\end{tabular}

HR: hazard ratio; CI: confidence interval; other abbreviations as in Table 1.

Figure 1 compares Kaplan-Meier curves for cardiac event-free survival between anemic and non-anemic patients (Log-rank $\mathrm{P}<0.001$ ). The incremental value of hemoglobin level in outcome prediction is shown in Figure 2. The basic clinical model consisted of the potential variables which were related to the adverse cardiac outcomes in the univariable analysis. These variables included age, body mass index, estimated glomerular filtration rate, chronic heart failure, and use of diuretics. The basic clinical model could significantly predict the adverse cardiac events (Chi-square $=44.149, \mathrm{P}<0.001)$. The addition of LVEF, LVMI, and E/Ea to the basic clinical model could significantly improve the prediction of adverse cardiac events $(\mathrm{P}<0.001)$. When hemoglobin was added to the final prediction model including basic clinical model, LVEF, LVMI, and E/Ea, it could significantly improve the prediction of adverse cardiac events $(\mathrm{P}=0.010)$.

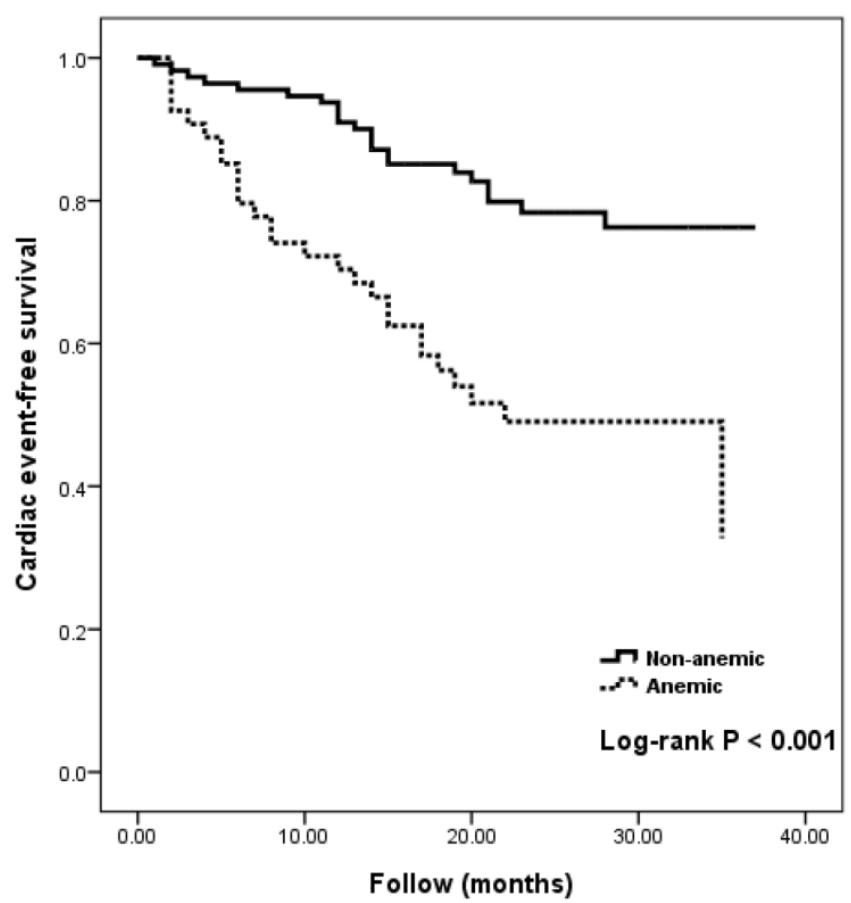

Figure 1. Results of Kaplan-Meier analysis of cardiac event-free survival in anemic and non-anemic patients.

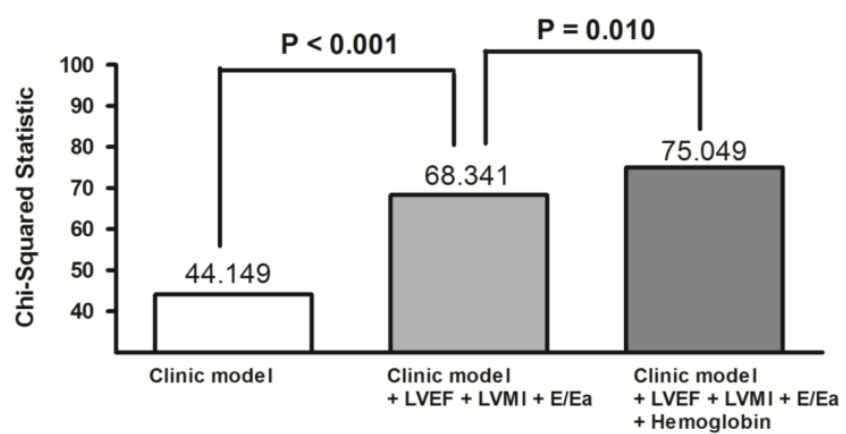

Figure 2. Value in predicting adverse cardiac events was significantly $(P=$ 0.010 ) improved by including hemoglobin in a Cox model that combined the basic clinical model (age, body mass index, estimated glomerular filtration rate, chronic heart failure, and diuretic use) with left ventricular ejection fraction (LVEF), left ventricular mass index (LVMI), and the ratio of early mitral inflow velocity to early diastolic mitral annulus velocity (E/Ea).

\section{Discussion}

This study evaluated hemoglobin for associations with cardiac outcomes in AF patients. Statistical analyses revealed that decreased hemoglobin was independently associated with increased cardiac events in the AF patients in this study. Inclusion of serum hemoglobin value significantly increased prognostic value compared to the combination of conventional clinical parameters and echocardiographic parameters.

Anemia is an independent predictor of adverse cardiovascular outcomes in patients with various cardiovascular diseases $[8,9,33]$. In patients with chronic heart failure, anemia is associated with increased severity of symptoms and increased mortality [34]. Treatment with erythropoietin and/or iron supplements can improve exercise tolerance, symptoms, and clinical outcomes in anemic patients with chronic heart failure [34-36]. These managements are associated with improvements in LVEF, New York Heart Association class, plasma B-type natriuretic peptide level, renal function, days of hospitalization, and required dose of diuretics [35-37]. The Atherosclerosis Risk in Communities cohort study of patients with coronary heart disease has also revealed that anemia has an independent association with increased risk (HR 1.41) of poor cardiovascular outcome [8]. However, the effect of blood transfusion in anemic patients 
with acute coronary syndrome is complex and controversial [38-40].

Several studies have investigated the relationship between anemia and mortality in patients with AF. In elderly patients with AF, Sharma et al. demonstrated that hematocrit level is an independent predictor of all-cause mortality [14]. An analysis of data in the AFCAS (Atrial Fibrillation undergoing Coronary Artery Stenting) registry by Puurunen et al. revealed that, in AF patients who undergo percutaneous coronary intervention, major adverse cardiac, cerebrovascular, and bleeding events are more likely in those with anemia compared to those without anemias [41]. The 1-year follow-up data in the AFCAS registry further revealed that anemia was an independent predictor of all-cause mortality (HR 1.62). Our study similarly showed that anemia is a predictor of adverse cardiac events in AF patients. Additionally, left ventricular systolic and diastolic dysfunction [22, 42, 43,44-46], and left ventricular hypertrophy [47] were significantly associated with high risks of cardiovascular morbidity and mortality in patients with AF. After adjustment for these essential echocardiographic parameters in the present study, serum hemoglobin was still an independent predictor of poor cardiac outcome in AF patients. Furthermore, in a Cox model consisting of the basic clinical model, LVEF, LVMI, and E/Ea, improvement in predicting poor cardiac prognosis in these patients was further increased by including hemoglobin. Hence, even when the conventional clinical and echocardiographic parameters are known, including serum hemoglobin further improves value in predicting cardiac outcome in AF patients.

Anemia is a risk factor in cardiovascular outcome for several reasons. First, chronic anemia is associated with left ventricular hypertrophy and heart failure. Patients with chronic anemia and hemoglobin less than $10 \mathrm{~g} / \mathrm{dL}$ exhibit several hemodynamic compensatory responses, including high cardiac output, low systemic vascular resistance, sodium and water retention, and reduction of renal blood flow and glomerular filtration rate. These responses may lead to increased cardiac workload and, consequently, left ventricular remodeling [48-50]. An abnormal left ventricular geometry may cause chronic heart failure and increase mortality risk. In the Randomized Etanercept North American Strategy to Study Antagonism of Cytokines (RENAISSANCE) trial, a 1 $\mathrm{g} / \mathrm{dL}$ increase in hemoglobin was associated with a $4.1 \mathrm{~g} / \mathrm{m}^{2}$ decrease in left ventricular mass and with a $15.8 \%$ reduction in mortality risk over a 24 -week period [51]. Second, anemia is also a risk factor for myocardial ischemia in patients with artherosclerosis and a mortality predictor in patients with acute coronary syndrome $[8,12]$. In the Atherosclerosis Risk in Communities (ARIC) cohort study, anemia with atherosclerosis was associated with increased risks of cardiovascular disease (HR 1.41) and all-cause mortality (HR 1.65) [8]. In a meta-analysis that included 27 studies, anemia was associated with increased all-cause mortality risk (HR 1.49) in patients with acute coronary syndrome [12]. The present study further found that low hemoglobin is a useful parameter for predicting adverse cardiac events in AF patients after adjusting for important clinical and echocardiographic parameters. Therefore, serum hemoglobin value should be measured in AF patients to improve prognostic value.

\section{Study limitations}

Most patients in this study received antihypertensive, antiplatelet, and anticoagulant medications for chronic conditions. For ethical reasons, these medications could not be withdrawn. Hence, their effects could not be excluded from this analysis. However, the use of medications was considered in the multivariable analysis. Since the subjects of this study were already being evaluated for heart disease by echocardiography, the generalizability of our conclusions is limited by the potential for selection bias. Other noted limitations are the large number of variables and the small number (49) of outcomes.

\section{Conclusions}

In patients with $\mathrm{AF}$, hemoglobin is a useful parameter for predicting adverse cardiac events and improves prognostic value when combined with conventional clinical and echocardiographic parameters.

\section{Competing Interests}

The authors have declared that no competing interest exists.

\section{References}

1. Go AS, Hylek EM, Phillips KA, et al. Prevalence of diagnosed atrial fibrillation in adults: National implications for rhythm management and stroke prevention: The anticoagulation and risk factors in atrial fibrillation (ATRIA) study. JAMA 2011;285: 2370-2375.

2. Fuster V, Ryden LE, Cannom DS, et al. ACC/AHA/ESC 2006 guidelines for the management of patients with atrial fibrillation: A report of the american college of cardiology/american heart association task force on practice guidelines and the european society of cardiology committee for practice guidelines (writing committee to revise the 2001 guidelines for the management of patients with atrial fibrillation): Developed in collaboration with the european heart rhythm association and the heart rhythm society. Circulation 2006;114: e257-354.

3. Wolf PA, Abbott RD, Kannel WB. Atrial fibrillation as an independent risk factor for stroke: The framingham study. Stroke 1991;22: 983-988.

4. McManus DD, Hsu G, Sung SH, et al. Atrial fibrillation and outcomes in heart failure with preserved versus reduced left ventricular ejection fraction. J Am Heart Assoc 2013;2: e005694.

5. Stewart S, Hart CL, Hole DJ, et al. A population-based study of the long-term risks associated with atrial fibrillation: 20-year follow-up of the Renfrew/Paisley study. Am J Med 2002;113: 359-364. 
6. Kim $\mathrm{MH}$, Johnston $\mathrm{SS}, \mathrm{Chu} \mathrm{BC}$, et al. Estimation of total incremental health care costs in patients with atrial fibrillation in the united states. Circ Cardiovasc Qual Outcomes 2011;4: 313-320.

7. McLean E, Cogswell M, Egli I, et al. Worldwide prevalence of anaemia, who vitamin and mineral nutrition information system, 1993-2005. Public Health Nutr 2009;12: 444-454.

8. Sarnak MJ, Tighiouart $H$, Manjunath $G$, et al. Anemia as a risk factor for cardiovascular disease in the atherosclerosis risk in communities (ARIC) study. J Am Coll Cardiol 2002;40: 27-33.

9. Groenveld HF, Januzzi JL, Damman K, et al. Anemia and mortality in heart failure patients a systematic review and meta-analysis. J Am Coll Cardiol 2008;52: 818-827.

10. Weiner DE, Tighiouart H, Vlagopoulos PT, et al. Effects of anemia and left ventricular hypertrophy on cardiovascular disease in patients with chronic kidney disease. J Am Soc Nephrol 2005;16: 1803-1810.

11. Mehdi U, Toto RD. Anemia, diabetes, and chronic kidney disease. Diabetes Care 2009:32: 1320-1326.

12. Lawler PR, Filion KB, Dourian T, et al. Anemia and mortality in acute coronary syndromes: A systematic review and meta-analysis. Am Heart J 2013;165: 143-153 e145.

13. Takabayashi $\mathrm{K}$, Unoki $\mathrm{T}$, Ogawa $\mathrm{H}$, et al. Clinical characteristics of atrial fibrillation patients with anemia: From the fushimi af registry. Eur Heart J 2013;34 (suppl 1): P389.

14. Sharma S, Gage BF, Deych E, et al. Anemia: An independent predictor of death and hospitalizations among elderly patients with atrial fibrillation. Am Heart J 2009;157: 1057-1063.

15. Hamdan A, Shapira $Y$, Bengal T, et al. Tissue doppler imaging in patients with advanced heart failure: Relation to functional class and prognosis. J Heart Lung Transplant 2006;25: 214-218.

16. Dokainish $\mathrm{H}$, Abbey $\mathrm{H}$, Gin $\mathrm{K}$, et al. Usefulness of tissue doppler imaging in the diagnosis and prognosis of acute right ventricular infarction with inferior wall acute left ventricular infarction. Am J Cardiol 2005;95: 1039-1042.

17. Chen SC, Chang JM, Liu WC, et al. Echocardiographic parameters are independently associated with increased cardiovascular events in patients with chronic kidney disease. Nephrol Dial Transplant 2012;27: 1064-1070.

18. Chang JM, Chen SC, Huang JC, et al. Anemia and left ventricular hypertrophy with renal function decline and cardiovascular events in chronic kidney disease. Am J Med Sci 2013;347: 183-189.

19. Ersboll M, Andersen MJ, Valeur N, et al. The prognostic value of left atrial peak reservoir strain in acute myocardial infarction is dependent on left ventricular longitudinal function and left atrial size. Circ Cardiovasc Imaging 2013:6: 26-33.

20. Motoki H, Borowski AG, Shrestha $K$, et al. Incremental prognostic value of assessing left ventricular myocardial mechanics in patients with chronic systolic heart failure. J Am Coll Cardiol 2012;60: 2074-2081.

21. Su HM, Lin TH, Hsu PC, et al. Association of interankle systolic blood pressure difference with peripheral vascular disease and left ventricular mass index. Am J Hypertens 2014;27: 32-37.

22. Su HM, Lin TH, Hsu PC, et al. Global left ventricular longitudinal systolic strain as a major predictor of cardiovascular events in patients with atrial fibrillation. Heart 2013;99: 1588-1596.

23. Hsu PC, Lee WH, Chu CY, et al. The ratio of early mitral inflow velocity to global diastolic strain rate as a useful predictor of cardiac outcomes in patients with atrial fibrillation. J Am Soc Echocardiogr 2014;27:717-725.

24. Devereux RB, Alonso DR, Lutas EM, et al. Echocardiographic assessment of left ventricular hypertrophy: Comparison to necropsy findings. Am J Cardiol 1986;57: 450-458

25. Lang RM, Bierig M, Devereux RB, et al. Recommendations for chamber quantification: A report from the american society of echocardiography's guidelines and standards committee and the chamber quantification writing group, developed in conjunction with the european association of echocardiography, a branch of the european society of cardiology. J Am Soc Echocardiogr 2005;18: 1440-1463.

26. Govindan M, Kiotsekoglou A, Saha SK, et al. Validation of echocardiographic left atrial parameters in atrial fibrillation using the index beat of preceding cardiac cycles of equal duration. J Am Soc Echocardiogr 2011;24: 1141-1147.

27. Lee CS, Lin TH, Hsu PC, et al. Measuring left ventricular peak longitudinal systolic strain from a single beat in atrial fibrillation: Validation of the index beat method. J Am Soc Echocardiogr 2012;25: 945-952.

28. Kusunose K, Yamada H, Nishio S, et al. Index-beat assessment of left ventricular systolic and diastolic function during atrial fibrillation using myocardial strain and strain rate. J Am Soc Echocardiogr 2012;25: 953-959.

29. Sohn DW, Song JM, Zo JH, et al. Mitral annulus velocity in the evaluation of left ventricular diastolic function in atrial fibrillation. J Am Soc Echocardiogr 1999;12: 927-931.

30. Wang CL, Lin $\mathrm{KH}$, Luqman $\mathrm{N}$, et al. Refinement on single-beat determination of left ventricular systolic function in patients with atrial fibrillation. J Am Soc Echocardiogr 2005;18: 913-918.

31. Govindan M, Kiotsekoglou A, Saha SK, et al. Validation of echocardiographic left atrial parameters in atrial fibrillation using the index beat of preceding cardiac cycles of equal duration. J Am Soc Echocardiogr 2011;24: 1141-1147.

32. World health organization. Hhaemoglobin concentrations for the diagnosis of anaemia and assessment of severity. Vitamin and Mineral Nutrition Information System. 2011:1-6.
33. Brown DW, Giles WH, Croft JB. Hematocrit and the risk of coronary heart disease mortality. Am Heart J. 2001;142:657-663.

34. Kotecha D, Ngo K, Walters JA, et al. Erythropoietin as a treatment of anemia in heart failure: Systematic review of randomized trials. Am Heart J. 2011;161:822-831 e822.

35. Silverberg DS, Wexler D, Blum M, et al. The use of subcutaneous erythropoietin and intravenous iron for the treatment of the anemia of severe, resistant congestive heart failure improves cardiac and renal function and functional cardiac class, and markedly reduces hospitalizations. J Am Coll Cardiol. 2000;35:1737-1744

36. Bolger AP, Bartlett FR, Penston HS, et al. Intravenous iron alone for the treatment of anemia in patients with chronic heart failure. J Am Coll Cardiol. 2006;48:1225-1227.

37. Palazzuoli A, Silverberg D, Iovine F, et al. Erythropoietin improves anemia exercise tolerance and renal function and reduces b-type natriuretic peptide and hospitalization in patients with heart failure and anemia. Am Heart J. 2006;152:1096 e1099-1015.

38. Wu WC, Rathore SS, Wang Y, et al. Blood transfusion in elderly patients with acute myocardial infarction. N Engl J Med. 2001;345:1230-1236.

39. Nikolsky E, Mehran R, Sadeghi HM, et al. Prognostic impact of blood transfusion after primary angioplasty for acute myocardial infarction: Analysis from the cadillac (controlled abciximab and device investigation to lower late angioplasty complications) trial. JACC Cardiovasc Interv. 2009;2:624-632.

40. Jolicoeur EM, O'Neill WW, Hellkamp A, et al. Transfusion and mortality in patients with st-segment elevation myocardial infarction treated with primary percutaneous coronary intervention. Eur Heart J. 2009;30:2575-2583.

41. Puurunen M, Kiviniemi T, Nammas W, et al. Impact of anaemia on clinical outcome in patients with atrial fibrillation undergoing percutaneous coronary intervention: Insights from the afcas registry. BMJ Open. 2014;4:e004700.

42. Dries DL, Exner DV, Gersh BJ, et al. Atrial fibrillation is associated with an increased risk for mortality and heart failure progression in patients with asymptomatic and symptomatic left ventricular systolic dysfunction: A retrospective analysis of the solvd trials. Studies of left ventricular dysfunction. J Am Coll Cardiol. 1998;32:695-703.

43. McMurray JJ, Ezekowitz JA, Lewis BS, et al. Left ventricular systolic dysfunction, heart failure, and the risk of stroke and systemic embolism in patients with atrial fibrillation: Insights from the aristotle trial. Circ Heart Fail. 2013;6:451-460.

44. Kosiuk J, Van Belle Y, Bode K, et al. Left ventricular diastolic dysfunction in atrial fibrillation: Predictors and relation with symptom severity. J Cardiovasc Electrophysiol. 2012;23:1073-1077.

45. Hogg K, Swedberg K, McMurray J. Heart failure with preserved left ventricular systolic function; epidemiology, clinical characteristics, and prognosis. J Am Coll Cardiol. 2004;43:317-327.

46. Olsson LG, Swedberg $\mathrm{K}$, Ducharme $\mathrm{A}$, et al. Atrial fibrillation and risk of clinical events in chronic heart failure with and without left ventricular systolic dysfunction: Results from the candesartan in heart failure-assessment of reduction in mortality and morbidity (CHARM) program. J Am Coll Cardiol. 2006;47:1997-2004.

47. Badheka AO, Shah N, Grover PM, et al. Outcomes in atrial fibrillation patients with and without left ventricular hypertrophy when treated with a lenient rate-control or rhythm-control strategy. Am J Cardiol. 2014:113:1159-1165.

48. Metivier F, Marchais SJ, Guerin AP, et al. Pathophysiology of anaemia: Focus on the heart and blood vessels. Nephrol Dial Transplant. 2000;15 Suppl 3:14-18

49. Anand IS, Chandrashekhar Y, Ferrari R, et al. Pathogenesis of oedema in chronic severe anaemia: Studies of body water and sodium, renal function, haemodynamic variables, and plasma hormones. British heart journal. 1993:70:357-362

50. Tang YD, Katz SD. Anemia in chronic heart failure: Prevalence, etiology, clinical correlates, and treatment options. Circulation. 2006;113:2454-2461.

51. Anand I, McMurray JJ, Whitmore J, et al. Anemia and its relationship to clinical outcome in heart failure. Circulation. 2004;110:149-154. 